\section{Cobertura por plano de saúde ou cartão de desconto: inquérito domiciliar na área de abrangência da Estratégia Saúde da Família}

\author{
Coverage by health insurance or discount cards: \\ a household survey in the coverage area of the \\ Family Health Strategy
}

\section{Cobertura por seguro de salud o tarjeta de descuento: encuesta domiciliaria en un área cubierta por la Estrategia Salud de la Familia}

Leonardo Ferreira Fontenelle 1

Maria Beatriz Junqueira de Camargo 2

Andréa Dâmaso Bertoldi 1

Helen Gonçalves 1

Ethel Leonor Noia Maciel 3

Aluísio J. D. Barros 1

\section{Resumo}

Este estudo foi desenhado para avaliar a cobertura por plano de saúde e seus motivos em uma população coberta pela Estratégia Saúde da Família. Nesta análise, descrevemos a cobertura por plano de saúde, total e por tipos, e analisamos sua associação com características de saúde e sociodemográficas. Entre os 31,3\% (IC95\%: 23, 8-39,9) de pessoas que relatavam cobertura por "plano de saúde”, 57,0\% (IC95\%: 45,2-68,0) estavam cobertos por cartões de desconto, que não oferecem qualquer tipo de cobertura para assistência médica, apenas descontos em farmácias, clínicas e hospitais. Tanto no caso dos planos de saúde quanto no dos cartões de desconto, os motivos para cobertura mais frequentemente relatados foram "para a segurança" e "para ter melhor atendimento". Ambas as coberturas se associaram à idade (65+ versus 15-24 anos: odds ratio ajustada, ORa = 2,98; IC95\%: 1,28-6,90; e ORa =3,67; IC95\%: 2,226,07, respectivamente) e ao nível econômico (desvio padrão adicional: $\mathrm{ORa}=$ 2,25; IC95\%: 1,62-3,14; e ORa =1,96; IC95\%: 1,34-2,97). Além disso, a cobertura por plano de saúde se associou à escolaridade (ORa = 7,59; IC95\%: 4,44-13,00) para Ensino Superior completo e ORa = 3,74 (IC95\%: 1,61-8,68) para Ensino Médio completo, em comparação a menos do que o Ensino Fundamental completo. Por outro lado, nem a cobertura por plano de saúde nem a por cartão de desconto se mostraram associadas ao estado de saúde ou ao número de doenças diagnosticadas. Em conclusão, estudos que pretendam avaliar a cobertura por saúde suplementar deveriam ser planejados de forma a poderem distinguir entre cartões de desconto e planos de saúde formais.

Saúde Suplementar; Seguro Saúde; Assistência à Saúde; Características da População

\author{
Correspondência \\ L. F. Fontenelle \\ Programa de Pós-graduação em Epidemiologia, Universidade \\ Federal de Pelotas. \\ Rua Marechal Deodoro 1160, 3o piso, Pelotas, RS 96020-220, \\ Brasil. \\ leonardof@leonardof.med.br \\ 1 Programa de Pós-graduação em Epidemiologia, \\ Universidade Federal de Pelotas, Pelotas, Brasil. \\ 2 Faculdade de Odontologia, Universidade Federal de Pelotas, \\ Pelotas, Brasil. \\ 3 Centro de Ciências da Saúde, Universidade Federal do \\ Espírito Santo, Vitória, Brasil.
}




\section{Introdução}

O Sistema Único de Saúde (SUS) avançou significativamente desde a sua criação, mas ainda sofre problemas graves de gestão. Os serviços de atenção à saúde são mal integrados entre si, e a força de trabalho da atenção primária à saúde tem uma elevada rotatividade. Além disso, desde a sua criação, o SUS recebe um financiamento inferior àquele inicialmente previsto. O resultado desses problemas é que, em 2008, apenas 56\% dos atendimentos médicos foram financiados pelo SUS; 19\% foram financiados diretamente pelas pessoas, e 25\%, por meio de planos de saúde 1 .

Os planos de saúde médico-hospitalares surgiram no Brasil, na década de 1940, e sua cobertura tem aumentado desde então 2,3,4. A Agência Nacional de Saúde Suplementar (ANS) monitora essa cobertura desde 2000, quando foi criada pela Lei Federal no 9.9615 para regular o setor. A ANS estima a cobertura por plano de saúde na forma da razão entre o número de beneficiários e o tamanho da população, de forma que seu numerador conta mais de uma vez as pessoas com cobertura múltipla. De junho de 2000 a junho de 2015, essa "razão de cobertura" por planos de saúde no Brasil aumentou de 18,6\% para 26,0\% (dados obtidos pelo sistema ANS Tabnet - http://www.ans.gov.br/anstabnet/).

A cobertura por plano de saúde é maior nas regiões Sul e Sudeste, nos municípios com maior porte populacional e nas áreas urbanas 6,7,8,9. Além disso, a cobertura por plano de saúde também aumenta conforme a idade 6,7,8,9 (com pouca diferença entre homens e mulheres 8 ) e o nível socioeconômico (indicado por escolaridade, tipo de ocupação, renda familiar ou índice de bens) 6,7,8,9,10,11,12,13. Já a relação entre a cobertura por plano de saúde e as condições de saúde não é tão clara. Apesar de um estudo qualitativo ter evidenciado a necessidade de assistência à saúde como explicação para a cobertura por plano de saúde 14 , inquéritos domiciliares têm encontrado maior cobertura por plano de saúde entre as pessoas com bom estado de saúde 8,12,13, e associação ausente ou de pequena magnitude dessa cobertura com a presença de doenças crônicas 8,11,12,13,15. Em análises ajustadas para idade e nível socioeconômico, a cobertura se mostrou discretamente maior entre as pessoas com bom estado de saúde em dois inquéritos 8,12, mas moderadamente menor em outro 13 .

Ao contrário dos planos de saúde, os cartões de desconto não oferecem propriamente uma cobertura para a assistência médico-hospitalar ou odontológica. O que os cartões de desconto oferecem, mediante mensalidades de baixo custo, são descontos em serviços de instituições ou profissionais conveniados 16,17. Tipicamente, esses descontos não se aplicam a procedimentos de alto custo, como internações hospitalares ou cirurgias, porque os cartões de desconto não são obrigados a uma lista mínima de procedimentos 16 . Isso acontece porque, apesar de competirem com os planos de saúde pelo mesmo mercado, os cartões de desconto não são regulados pela ANS 16,17. Ao contrário dos planos de saúde, os cartões de desconto não foram objeto de estudo epidemiológico até o momento, sendo que frequentemente inquéritos questionam sobre adesão a planos de saúde sem qualificá-los, de forma que cartões de desconto podem ser equivocadamente computados em estimativas de cobertura por plano de saúde.

O objetivo deste estudo foi descrever a prevalência e as características da cobertura por cartão de desconto e plano de saúde médico-hospitalar entre pessoas residentes em área de abrangência da Estratégia Saúde da Família (ESF), bem como analisar a associação entre essa cobertura e características de saúde e sociodemográficas.

\section{Métodos}

Este estudo foi baseado em um inquérito domiciliar realizado na zona urbana do Município de Pelotas, Rio Grande do Sul, Brasil. Foram incluídas, no estudo, pessoas com 15 anos de idade ou mais, residentes em áreas de abrangência da ESF. A ESF é o modelo prioritário para a organização da atenção primária à saúde no Brasil 18. Esse modelo é caracterizado por equipes multidisciplinares, cada uma delas responsável por uma área de abrangência com cerca de 3 mil pessoas 18 . Por sua vez, a área de abrangência de cada equipe é subdividida em microáreas, cada uma delas sob a responsabilidade de um agente comunitário de saúde (ACS) integrante da equipe 18. Cada unidade básica de saúde (UBS) pode abrigar uma ou mais equipes da ESF. 
O inquérito domiciliar foi realizado entre dezembro de 2007 e fevereiro de 2008, utilizando um delineamento amostral baseado em dados fornecidos pela Secretaria Municipal de Saúde de Pelotas. Na época, a zona urbana de Pelotas contava com 23 equipes da ESF, distribuídas entre 11 UBS. Quatro equipes da ESF foram excluídas da pesquisa: uma por ter sido utilizada no estudo piloto e outras três devido ao número reduzido de famílias. As 19 equipes restantes, distribuídas entre todas as 11 UBS, abrangiam um total de 101 microáreas. Dessas 101 microáreas, 30 foram excluídas por falta de ACS para colaborar com o inquérito, restando 81 microáreas. Os domicílios foram amostrados a partir dessas 81 microáreas, proporcionalmente ao número de domicílios cadastrados. O primeiro domicílio selecionado em cada microárea foi o domicílio seguinte a um ponto de partida definido por sorteio simples no cadastro mantido pelo ACS. Seguindo-se sempre da frente para o fundo dos terrenos e da direita para a esquerda do observador posicionado em frente aos quarteirões, os domicílios seguintes foram selecionados pulando-se cinco domicílios e selecionando-se o sexto, até os domicílios somarem o número de adultos e de idosos esperados para a amostra da microárea. Esse processo respeitou os limites geográficos das microáreas ao mesmo tempo que permitiu a inclusão de domicílios não cadastrados pelos ACS. Para cada domicílio selecionado, foram incluídas todas as pessoas residentes.

Cada pessoa foi abordada, ao menos, três vezes antes de ser considerada uma perda (por impossibilidade de contato) ou uma recusa. O único critério de exclusão da coleta de dados foi a incapacidade de participar da pesquisa, por exemplo, por surdez ou demência. $\mathrm{Na}$ análise deste artigo, foram excluídas ainda as pessoas com menos de 15 anos de idade, devido a não terem sido coletadas algumas das variáveis explanatórias.

Todas as variáveis do estudo foram obtidas a partir de um questionário abrangendo múltiplos domínios, incluindo demografia, nível econômico, condições de saúde e vários aspectos da interação com os sistemas de saúde público (SUS) e privado. Quando possível, os itens do questionário foram extraídos de outros inquéritos domiciliares, como a Pesquisa Nacional por Amostra de Domicílios (PNAD) 19. Mesmo assim, o questionário inicial foi aprimorado com base em um estudo piloto, adequando sua linguagem ao entendimento da população. Os dados coletados passaram por um extensivo controle de qualidade, incluindo digitação dupla, verificação de consistência interna dos dados e verificação presencial ou por telefone de trechos de $25 \%$ das entrevistas. As entrevistas com alguma inconsistência foram repetidas.

As variáveis explanatórias foram agrupadas em dois níveis de um modelo hierárquico de análise 20. No primeiro nível hierárquico, ficaram as variáveis sociodemográficas: faixa etária, sexo, cor da pele (autorrelatada e classificada em branca, parda e preta), situação conjugal, escolaridade e nível econômico (indicado por um índice baseado em bens, o Índice Econômico Nacional (IEN) 21, padronizado). No segundo nível hierárquico, ficaram as variáveis de condições de saúde: bom estado de saúde (autoavaliado) e número de diagnósticos médicos referidos (diabetes mellitus; hipertensão arterial; cardiopatia; doença cerebrovascular; ansiedade e/ou depressão; artropatia; lombalgia; câncer; asma ou doença pulmonar obstrutiva crônica).

A cobertura por plano de saúde foi definida para cada pessoa a partir da resposta à pergunta " $\mathrm{O}(\mathrm{a})$ senhor(a) é coberto(a) por plano de saúde?" e da listagem, pela pessoa, do nome dos "planos de saúde" pelos quais referia estar coberta. Cada "plano de saúde" foi classificado como cartão de desconto ou plano de saúde após investigação cuidadosa de cada empresa citada em relação aos contratos e coberturas oferecidos. A classificação foi realizada por três experts (profissionais que lidavam com faturamento em serviços de saúde suplementar), e as discrepâncias foram resolvidas pelo contato com as próprias organizações nomeadas. O critério para a classificação dos "planos de saúde" foi o pagamento dos serviços de saúde suplementar. Os planos foram classificados como cartão de desconto, no caso de desembolso direto (pagamento do próprio bolso), e como plano de saúde, no caso de pagamento pela operadora do plano de saúde, independente da existência de algum tipo de copagamento (frequentemente utilizado pelas operadoras como moderador de utilização). Depois de os "planos de saúde" terem sido classificados em planos de saúde propriamente ditos ou em cartões de desconto, a cobertura de cada pessoa foi categorizada como sem cobertura, cartão de desconto ou plano de saúde. Pessoas cobertas por um ou mais planos de saúde foram classificadas como cobertas por plano de saúde, mesmo que também estivessem cobertas por cartão de desconto.

Foi investigado também o principal motivo para a cobertura por "plano de saúde" (cartão de desconto ou plano de saúde propriamente dito), relatado livremente pelos participantes e codificado $a$ 
posteriori. O caráter empresarial foi classificado a partir da pergunta "Esse plano é pago por quem?". Os "planos de saúde" foram considerados empresariais, caso a pessoa respondesse "empresa (oferece)", em vez de "morador do domicílio" ou "outra pessoa que não mora no domicílio".

A associação entre as variáveis explanatórias e a cobertura por cartão de desconto ou plano de saúde foi, inicialmente, descrita por meio de proporções e, então, analisada por meio de razões de chances (odds ratios - OR) e seus intervalos de 95\% de confiança (IC95\%), obtidos por regressão logística multinomial simples. A regressão logística multinomial é uma extensão da regressão logística que admite variáveis de resposta com múltiplos níveis, não ordenados. As razões de chances estimadas pela regressão logística multinomial têm a mesma interpretação das OR estimadas por duas ou mais regressões logísticas, cada uma delas comparando um nível diferente da variável de resposta com o mesmo nível de referência da variável de resposta. No caso deste estudo, o nível de referência da variável de resposta foi a ausência de cobertura, e os outros níveis foram a cobertura por cartão de desconto e a cobertura por plano de saúde.

Depois da regressão logística multinomial simples, realizou-se a regressão logística multinomial múltipla. As variáveis do primeiro nível hierárquico (sociodemográficas) foram introduzidas simultaneamente e, então, eliminadas uma a uma, iniciando com aquela com o maior valor de p, até que todas as variáveis tivessem $\mathrm{p}<5 \%$. A seguir, foram introduzidas as variáveis do segundo nível hierárquico (condições de saúde), repetindo-se, entre essas, a eliminação para trás.

No caso do IEN padronizado e do número de diagnósticos médicos referidos, foi verificada a linearidade de sua associação com a cobertura por plano de saúde ou cartão de desconto. Para isso, foram introduzidos, no modelo de regressão, termos quadráticos cuja significância foi interpretada como evidência de fuga da linearidade.

O tamanho da amostra foi escolhido inicialmente com propósitos descritivos. Estimou-se que 422 idosos e 422 adultos mais jovens seriam o suficiente para estimar, em cada uma das faixas etárias, prevalências de $50 \%$ com uma margem de erro de 5 pontos percentuais, mesmo com uma proporção de recusas de até $10 \%$. Como nesta análise foram incluídos também os adolescentes com 15 anos de idade ou mais, a amostra foi ainda maior.

As análises foram realizadas com o software estatístico Stata 13 (StataCorp LP, College Station, Estados Unidos). O prefixo svy foi utilizado para corrigir as análises pelo efeito do delineamento amostral, considerando a UBS como unidade primária de análise e atribuindo pesos iguais a todas as observações, visto que foi utilizada uma amostra autoponderada.

O estudo foi aprovado pelo Comitê de Ética em Pesquisa da Faculdade de Medicina, Universidade Federal de Pelotas (ofício no 133/2006) e pela Secretaria Municipal de Saúde de Pelotas. Todos os participantes foram entrevistados apenas após a assinatura de um termo de consentimento livre e esclarecido.

\section{Resultados}

Durante o trabalho de campo, foram selecionados 550 domicílios, nos quais residiam 1.491 pessoas. Entre essas, houve 22 recusas, 13 exclusões por incapacidade de participar e duas perdas por impossibilidade de contato. Nesta análise, 369 pessoas não foram incluídas por terem menos de 15 anos de idade, de forma que a amostra consistiu em 1.085 pessoas. A idade mediana foi de 45 anos (variando de 15-95); houve predomínio de pessoas do sexo feminino (53,8\%), sem ensino fundamental completo $(68,2 \%)$ e que relatavam bom estado de saúde $(58,7 \%)$ (Tabela 1$)$.

A proporção de pessoas que relataram estar cobertas por plano de saúde foi de 31,3\% (IC95\%: 23,839,9); dentre essas pessoas, 57,0\% (IC95\%: 45,2-68,0) estavam cobertos exclusivamente por cartão de desconto. Assim, 12,7\% (IC95\%: 7,8-20,1) de todas as pessoas estavam cobertas por plano de saúde, e 16,9\% (IC95\%: 13,6-20,8), apenas por cartão de desconto. Essas duas proporções não somam 31,3\%, porque, para 26 participantes, não foi possível classificar, com segurança, a cobertura como sendo por cartão de desconto ou por plano de saúde.

Nem sempre a assistência à saúde era o principal produto do cartão de desconto. Dentre os sete cartões de desconto identificados, um deles era um benefício adicional para membros da respectiva associação, e outro era um benefício adicional a um serviço de assistência funerária. Identificamos 


\section{Tabela 1}

Características da amostra e distribuição da cobertura por cartão de desconto ou plano de saúde em pessoas com 15 anos de idade ou mais, residentes na área de abrangência da Estratégia Saúde da Família, na área urbana de Pelotas, Rio Grande do Sul, Brasil, 2008.

\begin{tabular}{|c|c|c|c|c|}
\hline & \multirow[b]{2}{*}{ Total (\%) } & \multicolumn{3}{|c|}{ Tipo de cobertura * } \\
\hline & & Sem cobertura (\%) & Cartão de desconto (\%) & Plano de saúde (\%) \\
\hline \multicolumn{5}{|l|}{ Idade (anos) } \\
\hline $15-24$ & $196(18,1)$ & $145(74,4)$ & $25(12,8)$ & $25(12,8)$ \\
\hline $25-44$ & $333(30,7)$ & $245(74,7)$ & $41(12,5)$ & $42(12,8)$ \\
\hline $45-64$ & $341(80,2)$ & $225(68,4)$ & $61(18,5)$ & $43(13,1)$ \\
\hline 65 ou mais & $215(18,8)$ & $130(62,8)$ & $52(25,1)$ & $25(12,1)$ \\
\hline \multicolumn{5}{|l|}{ Sexo } \\
\hline Masculino & $501(46,2)$ & $356(72,2)$ & $82(16,6)$ & $55(11,2)$ \\
\hline Feminino & $584(53,8)$ & $389(68,7)$ & $97(17,1)$ & $80(14,1)$ \\
\hline \multicolumn{5}{|l|}{ Situação conjugal } \\
\hline Casado & $665(61,4)$ & $441(67,7)$ & $123(18,9)$ & $87(13,4)$ \\
\hline Solteiro & $216(19,9)$ & $165(76,7)$ & $29(13,5)$ & $21(9,8)$ \\
\hline Separado & $74(6,8)$ & $59(80,8)$ & $7(9,6)$ & $7(9,6)$ \\
\hline Viúvo & $128(11,9)$ & $79(66,9)$ & $19(16,1)$ & $20(16,9)$ \\
\hline \multicolumn{5}{|l|}{ Cor da pele } \\
\hline Branca ** & $721(66,6)$ & $477(68,5)$ & $128(18,4)$ & $91(13,1)$ \\
\hline Parda & $166(15,3)$ & $128(77,1)$ & $22(13,2)$ & $16(9,6)$ \\
\hline Preta & $196(18,1)$ & $138(70,8)$ & $29(14,9)$ & $28(14,4)$ \\
\hline \multicolumn{5}{|l|}{ Escolaridade } \\
\hline Menos que o Ensino Fundamental completo & $735(68,2)$ & $537(75,3)$ & $117(16,4)$ & $59(8,3)$ \\
\hline Ensino Fundamental completo & $163(15,1)$ & $113(70,2)$ & $31(19,2)$ & $17(10,6)$ \\
\hline Ensino Médio completo & $161(14,9)$ & $85(53,5)$ & $28(17,6)$ & $46(28,9)$ \\
\hline Ensino Superior completo & $18(1,7)$ & $5(27,8)$ & $2(11,1)$ & $11(61,1)$ \\
\hline \multicolumn{5}{|l|}{ Quintis de nível econômico (IEN) } \\
\hline 1으 (mais pobre) & $217(20,0)$ & $184(86,4)$ & $19(8,9)$ & $10(4,7)$ \\
\hline 20 & $205(18,9)$ & $164(80,8)$ & $32(15,8)$ & $7(3,4)$ \\
\hline 3으 & $216(19,9)$ & $175(82,9)$ & $16(7,6)$ & $20(9,5)$ \\
\hline 4 은 & $212(19,5)$ & $122(58,9)$ & $50(24,1)$ & $35(16,9)$ \\
\hline 5o (mais rico) & $245(21,7)$ & $100(44,4)$ & $62(27,6)$ & $63(28,0)$ \\
\hline \multicolumn{5}{|l|}{ Número de doenças } \\
\hline Nenhuma & $328(30,2)$ & $230(71,4)$ & $50(15,5)$ & $42(13,0)$ \\
\hline $1-2$ & $461(42,5)$ & $324(71,2)$ & $72(15,8)$ & $59(13,0)$ \\
\hline $3-9$ & $296(27,3)$ & $191(67,7)$ & $57(20,2)$ & $34(12,1)$ \\
\hline \multicolumn{5}{|l|}{ Bom estado de saúde } \\
\hline Não & $448(41,3)$ & $312(71,2)$ & $78(17,8)$ & $48(11,0)$ \\
\hline Sim & $637(58,7)$ & $433(69,7)$ & $101(16,3)$ & $87(14,0)$ \\
\hline Total & 1.085 & $745(70,4)$ & $179(16,9)$ & $135(12,7)$ \\
\hline
\end{tabular}

IEN: Indicador Econômico Nacional.

* 26 participantes não puderam ter sua cobertura categorizada;

** Incluindo cor amarela (9) e indígenas (19).

ainda um cartão de desconto que, apesar de ter a assistência à saúde como principal produto, também mantinha convênio com academias de ginástica e com profissionais liberais fora da área da saúde.

Considerando tanto os cartões de desconto quanto os planos de saúde, os motivos para cobertura mais frequentemente relatados foram "para a segurança" (36,7\%; IC95\%: 28,6-45,7), "para ter melhor atendimento" (22,7\%; IC95\%: 15,5-31,9) e "porque a empresa oferece” (9,6\%; IC95\%: 5,1-17,3). Esse 
último motivo foi mais frequente entre as pessoas cobertas por plano de saúde (15,6\%; IC95\%: 7,829,0) do que entre as cobertas por cartão de desconto (5\%; IC95\%: 1,8-13,0) (p = 0,016). De fato, a proporção de "planos de saúde" empresariais (oferecidos pelo empregador) foi maior entre os planos de saúde propriamente ditos (36,5\%) do que entre os cartões de desconto (20,9\%).

Tanto a cobertura por plano de saúde quanto a cobertura por cartão de desconto aumentaram conforme o nível econômico (Tabela 2). A cobertura por cartão de desconto aumentou discretamente com a faixa etária, e a cobertura por plano de saúde aumentou com a escolaridade, de 8,3\% (IC95\%: 5,7-11,9), entre as pessoas sem o Ensino Fundamental completo, até 61,1\% (IC95\%: 30,5-84,9), entre aquelas com o ensino superior completo (OR = 20,0; IC95\%: 7,36-54,44) (Tabelas 1 e 2).

$\mathrm{Na}$ análise por regressão múltipla, a idade e o nível econômico se mostraram positivamente associados tanto à cobertura por plano de saúde quanto à cobertura por cartão de desconto (Tabela 3). A escolaridade se manteve associada apenas à cobertura por plano de saúde, com uma razão de chances ajustada de 7,59 (IC95\%: 4,44-13,00), para o Ensino Superior completo, e de 3,74 (IC95\%: 1,61-8,68), para o Ensino Médio completo, em comparação ao grupo com menos do que o Ensino Fundamental.

\section{Tabela 2}

Regressão logística multinomial simples da cobertura por cartão de desconto ou plano de saúde em pessoas com 15 anos de idade ou mais, residentes na área de abrangência da Estratégia Saúde da Família, na área urbana de Pelotas, Rio Grande do Sul, Brasil, 2008.

\begin{tabular}{|c|c|c|c|c|}
\hline & \multicolumn{4}{|c|}{ Tipo de cobertura } \\
\hline & \multicolumn{2}{|c|}{ Cartão de desconto } & \multicolumn{2}{|c|}{ Plano de saúde } \\
\hline & OR & IC95\% & OR & IC95\% \\
\hline \multicolumn{5}{|l|}{ Idade (anos) $[p=0,16]$} \\
\hline $15-24$ & 1,00 & - & 1,00 & - \\
\hline $25-44$ & 0,97 & $0,53-1,79$ & 0,99 & $0,64-1,54$ \\
\hline $45-64$ & 1,57 & $0,67-3,69$ & 1,11 & $0,73-1,67$ \\
\hline 65 ou mais & 2,32 & $1,46-3,68$ & 1,11 & $0,61-2,04$ \\
\hline \multicolumn{5}{|l|}{ Sexo $[p=0,38]$} \\
\hline Masculino & 1,00 & - & 1,00 & - \\
\hline Feminino & 1,08 & $0,84-1,39$ & 1,33 & $0,84-2,10$ \\
\hline \multicolumn{5}{|l|}{ Situação conjugal $[p=0,18]$} \\
\hline Casado & 1,00 & - & 1,00 & - \\
\hline Solteiro & 0,63 & $0,32-1,25$ & 0,64 & $0,43-0,96$ \\
\hline Separado & 0,42 & $0,17-1,04$ & 0,60 & $0,19-1,91$ \\
\hline Viúvo & 0,86 & $0,42-1,75$ & 1,28 & $0,84-1,95$ \\
\hline \multicolumn{5}{|l|}{ Cor da pele $[p=0,18]$} \\
\hline Branca & 1,00 & - & 1,00 & - \\
\hline Parda & 0,64 & $0,38-1,07$ & 0,65 & $0,38-1,14$ \\
\hline Preta & 0,78 & $0,38-1,59$ & 1,06 & $0,49-2,32$ \\
\hline \multicolumn{5}{|l|}{ Escolaridade $[p=0,02]$} \\
\hline Menos que o Ensino Fundamental & 1,00 & - & 1,00 & - \\
\hline Ensino Fundamental completo & 1,26 & $0,74-2,15$ & 1,37 & $0,85-2,20$ \\
\hline Ensino Médio completo & 1,51 & $0,77-2,96$ & 4,92 & $2,35-10,34$ \\
\hline Ensino Superior completo & 1,84 & $0,22-15,4$ & 20,0 & $7,36-54,44$ \\
\hline Nível econômico (IEN, + 1DP) [p < 0,001] & 1,90 & $1,35-2,67$ & 2,63 & $1,84-3,76$ \\
\hline Número de doenças $[p=0,68]$ & 1,05 & $0,93-1,18$ & 1,03 & $0,91-1,16$ \\
\hline \multicolumn{5}{|l|}{ Bom estado de saúde $[p=0,43]$} \\
\hline Não & 1,07 & $0,74-1,55$ & 0,77 & $0,50-1,16$ \\
\hline Sim & 1,00 & - & 1,00 & - \\
\hline
\end{tabular}

DP: desvio padrão; IC95\%: intervalo de 95\% de confiança; IEN: Indicador Econômico Nacional; OR: odds ratio. Nota: a categoria de referência da variável "tipo de cobertura" é "sem cobertura". 
Regressão logística multinomial múltipla da cobertura por cartão de desconto ou plano de saúde em pessoas com 15 anos de idade ou mais, residentes na área de abrangência da Estratégia Saúde da Família, na área urbana de Pelotas, Rio Grande do Sul, Brasil, 2008.

\begin{tabular}{|c|c|c|c|c|}
\hline & \multicolumn{4}{|c|}{ Tipo de cobertura } \\
\hline & \multicolumn{2}{|c|}{ Cartão de desconto } & \multicolumn{2}{|c|}{ Plano de saúde } \\
\hline & OR & IC95\% & OR & IC95\% \\
\hline \multicolumn{5}{|l|}{ Idade (anos) $[p=0,013]$} \\
\hline $15-24$ & 1,00 & - & 1,00 & - \\
\hline $25-44$ & 1,04 & $0,52-2,07$ & 1,09 & $0,61-1,92$ \\
\hline $45-64$ & 1,88 & $0,77-4,59$ & 1,71 & $0,77-3,76$ \\
\hline 65 ou mais & 3,67 & $2,22-6,07$ & 2,98 & $1,28-6,90$ \\
\hline \multicolumn{5}{|l|}{ Escolaridade $[p=0,004]$} \\
\hline Menos que o Ensino Fundamental & 1,00 & - & 1,00 & - \\
\hline Ensino Fundamental completo & 1,35 & $0,73-2,52$ & 1,25 & $0,61-2,57$ \\
\hline Ensino Médio completo & 1,43 & $0,66-3,10$ & 3,74 & $1,61-8,68$ \\
\hline Ensino Superior completo & 0,93 & $0,09-9,19$ & 7,59 & $4,44-13,0$ \\
\hline Nível econômico (IEN, + 1DP) [p = 0,001] & 1,96 & $1,34-2,97$ & 2,25 & $1,62-3,14$ \\
\hline
\end{tabular}

DP: desvio padrão; IC95\%: intervalo de 95\% de confiança; IEN: Indicador Econômico Nacional; OR: odds ratio.

Nota: a categoria de referência da variável "tipo de cobertura" é "sem cobertura".

Tanto na análise bruta (Tabela 2) quanto na ajustada, as variáveis relativas às condições de saúde não se mostraram associadas à cobertura por cartão de desconto ou por plano de saúde. Ter um diagnóstico médico a mais correspondeu a uma OR ajustada de 0,95 (IC95\%: 0,81-1,11), para cobertura por cartão de desconto, e de 1,10 (IC95\%: 0,95-1,28), para cobertura por plano de saúde (p = 0,19). Não ter um bom estado de saúde correspondeu a uma OR ajustada de 1,03 (IC95\%: 0,70-1,50), para cobertura por cartão de desconto, e de 0,91 (IC95\%: 0,56-1,49), para cobertura por plano de saúde (p=0,91).

A Figura 1 mostra como a cobertura por cartão de desconto e plano de saúde varia por grupos de escolaridade e por nível econômico (contínuo). Pela figura, vemos que indivíduos com escolaridade de Ensino Fundamental (completo ou incompleto) têm padrão muito similar. A cobertura por cartão de desconto diminui progressivamente entre indivíduos com Ensino Médio completo e Superior completo. De forma inversa, a cobertura por plano de saúde aumenta progressivamente com maior escolaridade. O efeito do nível econômico sobre a cobertura por cartão de desconto é bem mais marcado entre os dois grupos de escolaridade mais baixa. Entre indivíduos de Nível Superior completo, a cobertura por cartão de desconto se mantém baixa e estável. Sobre a cobertura de plano de saúde, o efeito de nível econômico é bem maior, sendo que a figura indica um aumento marcado de cobertura, passando, entre os indivíduos com Ensino Médio completo, de cerca de 20\%, para os de nível econômico na média da distribuição, para mais de $60 \%$, no topo da distribuição de riqueza.

\section{Discussão}

O principal achado deste inquérito domiciliar, realizado na área de abrangência da ESF da zona urbana de Pelotas, foi que $57 \%$ das pessoas que relatavam estar cobertas por plano de saúde estavam efetivamente cobertas por cartão de desconto. Tanto para os cartões de desconto quanto para os planos de saúde, os principais motivos para a cobertura mais frequentemente relatados foram "para a segurança" e "para melhor atendimento". Ambas as coberturas foram positivamente associadas à faixa etária e ao nível econômico (IEN), e nenhuma das coberturas se mostrou associada às características de saúde avaliadas (número de diagnósticos médicos referidos e estado de saúde autoavaliado). A escolaridade se associou positivamente à cobertura por plano de saúde e, como ilustrado na Figura 1, 
Figura 1

Cobertura por cartão de desconto ou plano de saúde conforme escolaridade e nível econômico (Índice Econômico Nacional - IEN).

1a) Sem cobertura

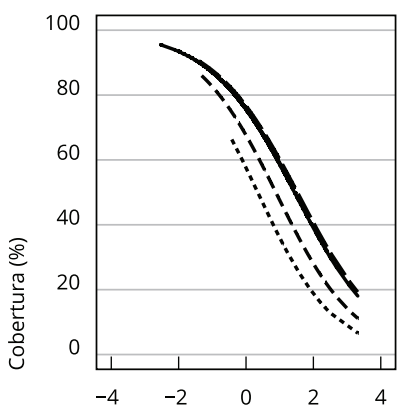

Nível econômico (padronizado) 1b) Cartão de desconto

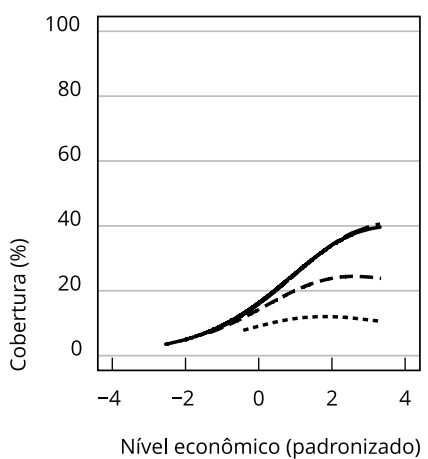

1c) Plano de saúde

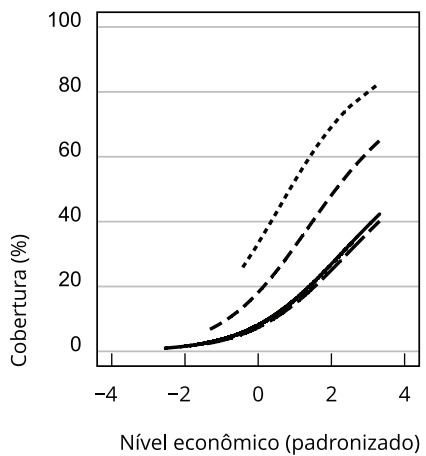

Escolaridade

- Menos que o ensino fundamental

----- Ensino médio completo

- Ensino fundamental completo

….. Ensino superior completo

as pessoas com maior escolaridade tiveram menor probabilidade de estarem cobertas por cartão de desconto ou de não estarem cobertas por qualquer uma das duas opções.

Os achados deste estudo sugerem que tanto os cartões de desconto quanto os planos de saúde tenham a sua cobertura explicada pela capacidade de pagamento das famílias (nível econômico), pela expectativa de necessitar da cobertura (faixa etária) e pela percepção do SUS como uma alternativa inferior (principais motivos relatados), mas não necessariamente pela efetiva necessidade atual (número de diagnósticos médicos, estado de saúde). Em linhas gerais, isso está de acordo com as conclusões de outros inquéritos realizados no Brasil 7,8,9,10,11,12, além de um estudo qualitativo 14 .

No caso específico dos planos de saúde, sua maior cobertura entre pessoas com maior escolaridade é um achado recorrente em estudos na área 6,7,8,10,11,12,13,22. A escolaridade é um importante indicador de nível socioeconômico e implica diretamente em ocupação e renda 23,24, que, por sua vez, estão fortemente associadas à cobertura por plano de saúde 8,9,10,12,13,25. Além disso, a escolaridade está positivamente associada a uma série de comportamentos saudáveis, como abster-se do tabagismo, ingerir frutas, verduras e legumes, praticar atividade física durante o lazer e passar por exames de rastreamento 6 . De fato, Bahia et al. 12 observaram que a escolaridade estava principalmente associada não à cobertura por plano de saúde empresarial, mas sim à cobertura por plano de saúde individual, o que sugere que a ocupação não seja a principal explicação para a maior cobertura entre pessoas com maior escolaridade. No presente estudo, como o nível econômico das famílias foi avaliado pelo IEN (um indicador composto que inclui a escolaridade do responsável pela família entre seus itens 21), é pouco provável que a renda familiar atual seja a principal explicação para a associação entre a escolaridade das pessoas e sua cobertura por plano de saúde. Dessa forma, associação entre escolaridade e cobertura por plano de saúde neste estudo é mais provavelmente explicada por uma combinação de (1) acesso a empregos formais e (2) diferentes prioridades com relação à seguridade em saúde. Note-se que, ao contrário da idade e do nível econômico, a escolaridade não se associou à cobertura por cartão de desconto. Seria desejável confirmar esse achado em inquéritos posteriores e explicá-lo por meio de estudos qualitativos.

No caso específico dos cartões de desconto, é possível que sua cobertura seja, em parte, explicada pela expectativa de necessitar de assistência funerária, e não apenas de assistência à saúde: neste estudo, o cartão de desconto com o maior número de pessoas cobertas era um benefício adicional a um plano funerário.

É digno de nota que nem a cobertura por plano de saúde nem a cobertura por cartão de desconto tenham guardado relação com as condições de saúde estudadas (estado de saúde e número de diag- 
nósticos médicos). As razões de chances estimadas neste estudo são semelhantes àquelas encontradas nas análises ajustadas de Bahia et al ${ }^{12}$, ao estudarem a cobertura por plano de saúde individual versus ausência de cobertura na PNAD 1998, e de Viacava et al. ${ }^{8}$, ao estudarem a cobertura por qualquer plano de saúde na Pesquisa Mundial de Saúde, de 2006. É provável, portanto, que a falta de significância estatística neste estudo se deva ao menor tamanho de sua amostra. De qualquer forma, tanto neste estudo quanto naqueles citados 8,12 , as razões de chance estimadas foram muito baixas, reforçando que as diferenças em cobertura por plano de saúde e cartão de desconto sejam explicadas principalmente por características predisponentes e facilitadoras, e não por características de necessidade. Já em um estudo da coorte de Bambuí, Lima-Costa et al. 13 relataram associação positiva entre a cobertura por plano de saúde e o estado de saúde, uma discordância que é possivelmente atribuível à diferença de faixa etária (a coorte de Bambuí é restrita a idosos). De qualquer forma, os resultados de Lima-Costa et al. 13 corroboram a noção de que a necessidade atual de assistência à saúde não seja, em geral, a principal explicação para a cobertura por plano de saúde.

Embora este inquérito domiciliar tenha sido desenhado especificamente para entender as estratégias de busca e utilização de serviços por uma população coberta pela ESF, ele também incluiu um levantamento detalhado sobre planos de saúde, oferecendo uma perspectiva única sobre os tipos de cobertura. O inquérito domiciliar foi representativo da população de estudo, graças não apenas ao seu plano amostral, mas também à sua baixa proporção de perdas e recusas e ao seu extensivo controle de qualidade. No entanto, assim como em outros estudos epidemiológicos e inquéritos populacionais, os dados deste inquérito domiciliar foram relatados pelas pessoas entrevistadas, as quais poderiam incorrer em erro de informação. É provável, portanto, que, em algum grau, os detalhes da cobertura por plano de saúde tenham escapado aos entrevistados.

Os resultados deste estudo são provavelmente generalizáveis para o resto do país, pois existem cartões de desconto em outros estados 16,17. A realização deste estudo em um único município não é necessariamente um problema, já que outros estudos realizados nesse mesmo município de porte médio têm encontrado resultados semelhantes aos de estudos de abrangência nacional 26,27,28. Além disso, acreditamos que a realização deste estudo na área de abrangência da ESF não tenha influenciado os resultados quanto aos motivos para a adesão a cartão de desconto e plano de saúde, que eram o nosso principal objetivo nessa análise. Essa suposição é reforçada pela semelhança entre a amostra e a população do município quanto à cobertura por plano de saúde e à proporção de planos de saúde empresariais. Utilizando a população estimada pelo Instituto Brasileiro de Geografia e Estatística (IBGE) para o município em 2007 e o número de vínculos de plano de saúde no município em setembro do mesmo ano, de acordo com a ANS, estima-se uma razão de cobertura de 13,3\%, frente à proporção de cobertura de 12,7\% neste estudo. Além disso, calcula-se, a partir desses dados da ANS, que 40,2\% dos vínculos de plano de saúde sejam empresariais, frente a 36,5\% neste estudo.

\section{Conclusão}

Pelo que sabemos, este foi o primeiro estudo epidemiológico capaz de diferenciar cartões de desconto de planos de saúde. Ao falhar em fazer essa distinção, é presumível que estudos anteriores tenham tido suas estimativas de cobertura por plano de saúde superestimadas. Também é presumível que as associações descritas em estudos anteriores reflitam mais propriamente o conjunto de cartão de desconto e plano de saúde do que um ou outro. Isso sugere ser importante que novos estudos sobre a cobertura por plano de saúde tenham seus questionários explicitamente construídos para poderem diferenciar os dois tipos de cobertura. Somente desse modo, será possível compreender melhor tanto as implicações da cobertura por plano de saúde quanto aquelas da cobertura por cartão de desconto. 


\section{Colaboradores}

L. F. Fontenelle definiu o objetivo do estudo, analisou os dados, discutiu os resultados e rascunhou o manuscrito. M. B. J. Camargo colaborou na análise dos dados e na discussão dos resultados, e revisou o manuscrito. A. D. Bertoldi e H. Gonçalves planejaram e coordenaram a coleta de dados, colaborou na discussão dos resultados e revisou o manuscrito. E. L. N Maciel colaborou na discussão dos resultados e revisou o manuscrito. A. J. D. Barros colaborou na definição do objetivo do estudo, no seu desenho, na análise dos dados e na discussão dos resultados, revisou o manuscrito e coordenou a coleta dos dados. Todos os autores aprovaram a versão final para publicação.

\section{Agradecimentos}

Os autores são gratos à Secretaria Municipal de Saúde de Pelotas pela valiosa colaboração no planejamento do trabalho de campo. E ao financiamento pelo Edital MCT/CNPq/MS-SCTIE-DECIT no 26 de 2006; Edital FAPERGS/MS/CNPq/SESRS PPSUS/RS 07/0078.2.

\section{Referências}

1. Paim J, Travassos C, Almeida C, Bahia L, Macinko J. The Brazilian health system: history, advances, and challenges. Lancet 2011; 377:1778-97.

2. Almeida C. O mercado privado de serviços de saúde no Brasil: panorama atual e tendências da assistência médica suplementar. Brasília: Instituto de Pesquisa Econômica Aplicada; 1998. (Texto para Discussão, 599).

3. Ocké-Reis CO. O Estado e os planos de saúde no Brasil. Revista do Serviço Público 2000; 51:124-47.

4. Bahia L. Planos privados de saúde: luzes e sombras no debate setorial dos anos 90. Ciênc Saúde Coletiva 2001; 6:329-39.

5. Presidência da República. Lei no 9.961 de 28 de Janeiro de 2000. Cria a Agência Nacional de Saúde Suplementar - ANS e dá outras providências. Diário Oficial da União 2000; 29 jan.

6. Malta DC, Oliveira MR, Moura EC, Silva SA, Zouain CS, Santos FP, et al. Fatores de risco e proteção para doenças crônicas não transmissíveis entre beneficiários da saúde suplementar: resultados do inquérito telefônico Vigitel, Brasil, 2008. Ciênc Saúde Coletiva 2011; 16:2011-22.

7. Pinto LF, Soranz DR. Planos privados de assistência à saúde: cobertura populacional no Brasil. Ciênc Saúde Coletiva 2004; 9:85-98.

8. Viacava F, Souza-Júnior PRB, Szwarcwald CL. Coverage of the Brazilian population 18 years and older by private health plans: an analysis of data from the World Health Survey. Cad Saúde Pública 2005; 21 Suppl 1:S119-28.

9. Instituto Brasileiro de Geografia e Estatística. Um panorama da saúde no Brasil: acesso e utilização dos serviços, condições de saúde e fatores de risco e proteção à saúde, 2008. Rio de Janeiro: Instituto Brasileiro de Geografia e Estatística; 2010.

10. Machado AF, Andrade MV, Maia AC. A relação entre estrutura ocupacional e acesso a plano de saúde no Brasil: uma análise para 1998 e 2003. Cad Saúde Pública 2012; 28:758-68.

11. Hernandes ESC, Lebrão ML, Duarte YAO, Santos JLF. Health insurance coverage of the elderly and socioepidemiological characteristics associated. Rev Saúde Pública 2012; 46:1030-8.

12. Bahia L, Costa AJL, Fernandes C, Luiz RR, Cavalcanti MLT. Segmentação da demanda dos planos e seguros privados de saúde: uma análise das informações da PNAD/98. Ciênc Saúde Coletiva 2002; 7:671-86.

13. Lima-Costa MFF, Guerra HL, Firmo JOA, Vidigal PG, Uchoa E, Barreto SM. The Bambui Health and Aging Study (BHAS): private health plan and medical care utilization by older adults. Cad Saúde Pública 2002; 18:177-86.

14. Farias LO. Estratégias individuais de proteção à saúde: um estudo da adesão ao sistema de saúde suplementar. Ciênc Saúde Coletiva 2001; 6:405-16. 
15. Barros MBA, César CLG, Carandina L, Tor re GD. Desigualdades sociais na prevalência de doenças crônicas no Brasil, PNAD-2003. Ciênc Saúde Coletiva 2006; 11:911-26.

16. Salvatori RT, Ventura CAA. A agência nacional de saúde suplementar - ANS: onze anos de regulação dos planos de saúde. Organizações \& Sociedade 2012; 19:471-88.

17. Santos FP, Malta DC, Merhy EE. A regulação na saúde suplementar: uma análise dos principais resultados alcançados. Ciênc Saúde Coletiva 2008; 13:1463-75.

18. Departamento de Atenção Básica, Secretaria de Atenção à Saúde, Ministério da Saúde. Política Nacional de Atenção Básica. Brasília: Ministério da Saúde; 2012. (Série E, Legislação em Saúde).

19. Travassos C, Viacava F, Laguardia J. Os suplementos saúde na Pesquisa Nacional por Amostra de Domicílios (PNAD) no Brasil. Rev Bras Epidemiol 2008; 11 Suppl 1:98-112.

20. Victora CG, Huttly SR, Fuchs SC, Olinto MT. The role of conceptual frameworks in epidemiological analysis: a hierarchical approach. Int J Epidemiol 1997; 26:224-7.

21. Barros AJD, Victora CG. A nationwide wealth score based on the 2000 Brazilian demographic census. Rev Saúde Pública 2005; 39:523-9.

22. Lima Costa MF. Estilos de vida e uso de serviços preventivos de saúde entre adultos filiados ou não a plano privado de saúde (inquérito de saúde de Belo Horizonte). Ciênc Saúde Coletiva 2004; 9:957-64.
23. Galobardes B, Shaw M, Lawlor DA, Lynch JW, Smith GD. Indicators of socioeconomic position (part 1). J Epidemiol Community Health 2006; 60:7-12.

24. Howe LD, Galobardes B, Matijasevich A, Gordon D, Johnston D, Onwujekwe O, et al. Measuring socio-economic position for epidemiological studies in low- and middle-income countries: a methods of measurement in epidemiology paper. Int J Epidemiol 2012; 41:871-86.

25. Farias LO, Melamed C. Segmentação de mercados da assistência à saúde no Brasil. Ciênc Saúde Coletiva 2003; 8:585-98.

26. Mendoza-Sassi R, Béria JU, Barros AJD. Outpatient health service utilization and associated factors: a population-based study. Rev Saúde Pública 2003; 37:372-8.

27. Camargo MBJ, Dumith SC, Barros AJD. Uso regular de serviços odontológicos entre adultos: padrões de utilização e tipos de serviços. Cad Saúde Pública 2009; 25:1894-906.

28. Dilélio AS, Tomasi E, Thumé E, Silveira DS, Siqueira FCV, Piccini RX, et al. Padrões de utilização de atendimento médico-ambulatorial no Brasil entre usuários do Sistema Único de Saúde, da saúde suplementar e de serviços privados. Cad Saúde Pública 2014; 30:2594-606. 


\section{Abstract}

This study was designed to assess the reasons for health insurance coverage in a population covered by the Family Health Strategy in Brazil. We describe overall health insurance coverage and according to types, and analyze its association with health-related and socio-demographic characteristics. Among the 31.3\% of persons (95\% CI: 23.8-39.9) who reported "health insurance" coverage, 57.0\% (95\%CI: 45.2-68.0) were covered only by discount cards, which do not offer any kind of coverage for medical care, but only discounts in pharmacies, clinics, and hospitals. Both for health insurance and discount cards, the most frequently cited reasons for such coverage were "to be on the safe side" and "to receive better care". Both types of coverage were associated statistically with age $(+65$ vs. 15-24 years: adjusted odds ratios, $a \mathrm{OR}=2.98$, 95\%CI: $1.28-6.90$; and $\mathrm{aOR}=3.67$; 95\%CI: $2.22-$ 6.07 , respectively) and socioeconomic status (additional standard deviation: $a O R=2.25,95 \% \mathrm{CI}$ : 1.62-3.14; and $a \mathrm{OR}=1.96,95 \% \mathrm{CI}: 1.34-2.97)$. In addition, health insurance coverage was associated with schooling ( $a \mathrm{OR}=7.59$, 95\% CI: 4.44-13.00) for complete University Education and $a \mathrm{OR}=$ 3.74 (95\% CI: 1.61-8.68) for complete Secondary Education, compared to less than complete Primary Education. Meanwhile, neither health insurance nor discount card was associated with health status or number of diagnosed diseases. In conclusion, studies that aim to assess private health insurance should be planned to distinguish between discount cards and formal health insurance.

Supplemental Health; Health Insurance; Delivery of Health Care; Population Characteristics

\section{Resumen}

Este estudio se diseñó para evaluar la cobertura por seguro de salud y sus causas en una población cubierta por la Estrategia Salud de la Familia. En este análisis, describimos la cobertura por seguro de salud, total y por tipos, y analizamos su asociación con características de salud y sociodemográficas. Dentro del 31,3\% (IC95\%: 23, 8-39,9) de personas que informaban contar con una cobertura por "seguro de salud" un 57,0\% (IC95\%: 45,2-68,0) estaban cubiertas por tarjetas de descuento, que no ofrecen cualquier tipo de cobertura para la asistencia médica, solamente descuentos en farmacias, clínicas y hospitales. Tanto en el caso de los seguros de salud, como en el de las tarjetas de descuento, los motivos de cobertura más frecuentemente relatados fueron "por seguridad" y "para tener una mejor atención". Ambas coberturas se asociaron a la edad (65+ versus 15-24 años: odds ratio ajustada, ORa = 2,98; IC95\%: 1,28-6,90; y ORa = 3,67; IC95\%: 2,22-6,07, respectivamente), y al nivel económico (desvío patrón adicional: ORa $=$ 2,25; IC95\%: 1,62-3, 14; y ORa = 1,96; IC95\%: 1,34-2,97). Además, la cobertura por seguro de salud se asoció a la escolaridad (ORa = 7,59; IC95\%: 4,44-13,00) para la Enseñanza Superior completa y ORa = 3,74 (IC95\%: 1,61-8,68) para el Nivel Medio completo, en comparación con los menores indices por la Enseñanza Fundamental completa. Por otro lado, ni la cobertura por seguro de salud, ni la por tarjeta de descuento, se mostraron asociadas al estado de salud o al número de enfermedades diagnosticadas. En conclusión, los estudios que pretendan evaluar la cobertura de seguro de salud privado se deberían planear de tal forma que puedan distinguir entre tarjetas de descuento y seguros de salud formales.

Salud Suplementaria; Seguro de Salud; Prestación de Atención de Salud; Características de la Población
Recebido em 31/Ago/2015

Versão final reapresentada em 19/Nov/2016 Aprovado em 13/Dez/2016 